\title{
Evaluating Internet Interviews With Gay Men
}

Russel Ayling, DClinPsy

University of Exeter, Devon, UK

(Now at Barts and the London NHS Trust, London, UK)

Contact details:

Clinical Psychology Service

Infection and Immunity Specialty Group

Ambrose King Centre

Royal London Hospital

LONDON E1 1BB

Tel +44 (0)207377 7038

Fax +44 (0)207377 7648

e-mail russel.ayling@bartsandthelondon.nhs.uk

Avril J Mewse, $\mathrm{PhD}$

University of Exeter, Devon, UK

Contact details:

School of Psychology

University of Exeter

Washington Singer Laboratories

Perry Road

EXETER EX4 4QG

Tel +44 (0)1392 264626

Fax: +44 (0)1392 264623

e-maila.j.mewse@exeter.ac.uk 


\begin{abstract}
We describe the use of the Internet to conduct online synchronous interviews on gay men's experience of Internet sex-seeking and barebacking, using interpretative phenomenological analysis (IPA). We then present the results of a separate IPA of the issues that we encountered during the research. The analysis is focused around four superordinate themes: positive experience, technological issues, boundaries and requests/expectations. We argue that online qualitative research is valuable in its own right, and that the advantages considerably outweigh the difficulties, particularly in accessing individuals who may not participate in other types of research settings, to discuss experiences that would otherwise be too difficult to talk about. Several issues that are problematic in more conventional research still apply, albeit in a different guise, but these must be situated in the context of who is being researched, what and why, and can be largely overcome with more creative methodologies and considered preparation.
\end{abstract}

Keywords

Gays and lesbians; HIV/AIDS; Internet; interpretive methods; psychology; psychotherapy; research, online; risk, behaviors; sexual health; sexuality 
Seven years ago, researchers were commenting that it was "perhaps surprising that the suitability of the Internet for conducting research remains relatively unexplored" (Mann \& Stewart, 2000, p. 4). Since then, the number of studies using Internet methodologies has increased greatly, although considerably more use has been made of the Internet for the collection of quantitative than qualitative data. Articles are emerging that document some of the issues concerning the use of the Internet for qualitative research, however, a convergence of opinion about the suitability of the Internet for research still appears to be some way off yet.

Several specialist resources are available to support the qualitative researcher in exploring non face-to-face interview methods (Chen \& Hall, 2003; Coombes, 2001; Fox, Morris \& Rumsey, 2007; Hamilton \& Bowers, 2006; Hewson, Yule, Laurent \& Vogle, 2003; Hunt \& McHale, 2007; Jones, 1999; Mann \& Stewart, 2000). These can be grouped into two main types: asynchronous and synchronous. Asynchronous methods do not require participants and researchers to be present at the same time, allowing "conversation" over a longer period of time. Most asynchronous online research uses e-mail, the researcher sending an initial set of questions to the participant, who replies at their convenience. The researcher then follows up on their responses in another e-mail, and so on. Group discussions can be facilitated in a similar way using mailing lists, bulletin boards and Internet forums. In synchronous methods, all parties converse together in real time. Examples of synchronous methods are telephone conversations, webcam based chat, and Internet text-based chat.

All of the methods described above have the advantage of reaching people who either cannot or will not attend face-to-face interviews, for reasons such as geographical distance, physical disability, social isolation, inconvenient timings, or simply embarrassment and unwillingness to meet a researcher in the flesh. Asynchronous interviews give researcher and 
participant more time to consider their questions and answers, which allows a different quality of discourse to that generated in real-time. There is a time saving in that each person only needs to be present for their portion of the conversation. Synchronous interviews allow an interaction that is more like an everyday face-to-face conversation. The degree of anonymity increases with the move from webcam (audio and visual material) to telephone (audio only) to text-based interactions. Increasing anonymity allows participants to be more open in their responses, particularly when discussing sensitive topics, allowing their "true self" to talk. Some researchers, however, are concerned that participants might willfully pretend to be someone that they are not.

Particularly in synchronous text-based interviews, conversations are slower, the material is shorter and is more condensed than in spoken interviews. However, data are transcribed automatically, which may balance out the extra time taken to interview, and the data are not subject to the transcriber's potential biases or errors. Text-based online interviews may also be more ecologically valid for interviewing those engaging in online behaviors or who are part of online communities. However, text-based communication can be affected by participant distraction and motivation, participant and researcher access to and competence with the necessary technology, and ability to communicate effectively without audio-visual cues. The privacy of the exchange is also of particular concern in all non face-to-face environments, and is more challenging to ascertain when using the Internet.

Fox et al. (2007) comment that "qualitative researchers who use novel methodological approaches should be prepared to engage in a process of reflection and reflexivity to make transparent the experience and demonstrate the viability of the method" and note that most published articles have concentrated on the methodological costs and benefits. In one of few 
methodological articles specifically commenting on the "validity" of data produced by online interviews, Davis, Bolding, Hart, Sherr, and Elford (2004, p. 951) frame synchronous text-based online interviews as a "textual performance" that is affected by social convention and technical limitation to produce data that are ambiguous and highly contextualized. They argue that this limits the extent to which this method can be used for an in-depth exploration of meaning or description of social or sexual performance and conclude that Internet qualitative methods should be used alongside, rather than as an alternative to, traditional face-to-face interviews.

In this article, we describe the use of the Internet to conduct online synchronous interviews in order to understand gay men's experience of Internet sex-seeking and barebacking $^{1}$, using interpretative phenomenological analysis (IPA). We then present the results of a separate IPA of the issues that both the participants and we as researchers encountered during the research process. We discuss these issues in the context of existing literature on online qualitative research interviewing, and make some recommendations for further research.

\section{Method}

Ethical approval for the research was obtained from the Ethics Committee of the School of Psychology, University of Exeter.

\section{Participants}

We recruited nine single gay men who believed themselves to be HIV-negative, and who had barebacked with at least one man they had found using the Internet. We recruited these men via advertisements in Gay Times $^{2}$ and on gay-oriented Internet personals websites. ${ }^{3} \mathrm{We}$ constructed a website where interested participants could access participant information and complete an online pro-forma to provide demographic details (such as age, location, occupation), times that they were available for interview, and informed consent. 


\section{Procedure}

Once participants had completed the webform and given informed consent, we contacted them to arrange an interview time and asked them to download the secure internet chat software. Two participants chose to use different non-encrypted software; we explained the data security implications of this to the participants, who gave informed consent to this choice. The first author carried out interviews using a standard semi-structured interview schedule comprising a small number of open-ended, non-directive questions informed by previous research focusing on barebacking and Internet sex-seeking. As we were aware of the tendency for Internet exchanges to be much more condensed than face-to-face interviews, we prepared several prompts for each question in case substantial answers were not forthcoming. Consistent with IPA methodology, the interviewer attempted to approach each interview as independent from any others, using the interview schedule as a guide to content but allowing the conversation to flow naturally, led by the participants wherever possible. We chose a reflective, probing approach to interviewing, concentrating on building rapport to allow participants to disclose their perspective. Interviews lasted between one and three hours, and we followed standard data protection procedures for electronic research data. The participant information and interview schedule are available from the authors.

\section{Ethical considerations}

Whilst we took all reasonable steps to maximize data security, any research using the Internet carries a level of risk that the data can be intercepted, and we made this clear to participants in order than they could make an informed choice to participate. By nature of the research topic and inclusion criteria, our participants had already engaged regularly in many explicit and sensitive conversations using the Internet, within a far less secure environment. In 
order to maximize the security of initial demographic data collected by the online pro-forma, this was hosted on a website exclusively hosted and maintained by the University of Exeter.

We chose the software X-IM (see http://www.X-im.net/ for specification) to perform the interviews. The software allows encrypted one-to-one text based chat, analogous to a conversation between two people on the telephone. The software required us to know the individual's e-mail address, therefore for maximum anonymity, we asked participants to use a free e-mail address, such as those provided by Hotmail or Yahoo. Outside the interview (for contacts about recruitment and participant validation of the analysis, for example), we communicated with participants using this e-mail address.

We kept e-mail addresses separate from interview data by means of a code, known only to the first author. Intentionally, we recorded few identifying details about participants and did not collect names or postal addresses. Participants' e-mails opting into the study, formed evidence of informed consent, which was revisited at the beginning of the interview. We removed any identifying details revealed during the interview from the data.

The X-IM software provided a transcript on each interview ready for analysis. The first author held these files on his computer, and secured both computer and files with a password. The computer was also protected with a firewall and up to date anti-virus software in order to keep out intruders and prevent virus transmission. The first author kept CD and paper copies of all data in a locked cabinet.

We did not expect the content of the interviews to be particularly distressing, so we followed standard procedures for the management of distress during or after research interviews. Before the interviews, we informed participants that there was a risk that talking about private and intense experiences might make them feel distressed or confused. We offered an online 
debrief to all participants, allowing them the opportunity to process their feelings about the interview. One of the questions in the debrief was whether the participant felt that they needed any kind of information, advice or therapy; we also provided details of organizations able to provide support, such as help lines for people worried about their sexuality, sexual health and HIV, NHS Direct (a nurse-led telephone service providing medical advice and interventions) and Samaritans (a volunteer-led national telephone service providing emotional support for those in crisis). Participants were given the opportunity to e-mail or telephone us at any point following the interview should they wish to do so, although a follow-up session where transcripts could be shared and further reflections made on their participation was not explicitly provided, and this was an omission.

Analytic Strategy

The chat software provided transcripts automatically and the first author changed the names of participants and people they identified. The first author analyzed the transcripts using IPA. IPA provides a qualitative approach that allows both observation of the meanings that participants attach to their behaviors and interpretation of the processes by which participants come to do so. It does so by grounding itself both in phenomenology (the personal perception or account of an object of event) and symbolic interaction (the meanings individuals ascribe to events obtained through a process of interpretation and social interactions). IPA allows the researcher to engage in dialogue with both social cognitive concepts such as beliefs, attitudes and behaviors, and discursive concepts such as the use of context and language (Smith, 1996).

Consistent with IPA methodology, the first author read each interview a number of times, paying particular attention to semantic content and language use, key words, phrases and explanations. He coded each with a key word or phrase, known as emergent themes, and flagged 
those that were repeated by the same participant, or different participants. These are known as recurrent themes and represent shared understandings. He generated a file of extracts containing all instances of each recurrent theme, and grouped similar recurrent themes into superordinate themes. For brevity, we have chosen the most articulate or powerful examples within each superordinate theme for purposes of illustration, with emphasis on similarity and consistency between participants, but we have highlighted instances of marked contradiction and polarity where they occurred. As a result, each theme provides a distinct representation of thoughts and feelings about an issue.

Consistent with IPA methodology, the first author attempted to suspend existing knowledge and experience (epoché), in order to see the world as experienced by participants. The first author is a clinical psychologist with specialist interest in psychodynamic psychotherapy and HIV/sexual health, and this interest influenced the interpretative level of the analysis, which is encouraged by IPA methodology. For purposes of validation, the second author read all scripts and checked that: themes were grounded in the data; representative; and constructed in a way that made intuitive sense. Three clinical health psychologists (one with considerable expertise in IPA) provided further validation that themes were consistent with their clinical experience. Participants were also invited to comment on a draft of the analysis although none chose to do so.

\section{Results of Initial Study}

The aim of the original research was to explore how gay men make sense of barebacking and to examine the role the Internet plays in this. The analysis was formed of three superordinate themes of physicality/emotionality, dominance/submission and engagement/avoidance of risk management. From these, two major findings emerged. The first 
was that participants are not seeking HIV infection and death; rather they are trying to take care of themselves whilst doing something they love that carries risk. This is a source of anxiety, uncertainty and conflict. The second was that the Internet provides a means for participants to manage this uncertainty and attempt to reduce the risk of HIV infection, although some of the strategies employed by a small number of participants were of questionable efficacy in terms of minimizing risk .

\section{Analysis}

For the current article, we have included in the analysis only the emergent themes that concern the participants' and researcher's experiences of engaging in the research. To communicate the data as authentically as possible, material from transcripts has been quoted verbatim, including non-standard typography.

In the analysis, we focus on four superordinate themes: positive experience, technological issues, boundaries and requests/expectations. We then consider these themes in the context of existing literature in the discussion section of this article.

\section{Superordinate Theme: Positive Experience}

This theme illustrates participants' experience of the research as useful and enjoyable, their thoughts on the interview style, feelings about their involvement with the research, and hopes for its outcome.

Participants were unanimous in their positive evaluation of the research interview. They used words like "good", "fun" and "fine" to describe their experience. Some noted that time had "flown by" and "I'm enjoying it. Do this again if you like".

Participants also commented on the interview style. One expressed his preference for interview over survey based methods "it was quite strange to have someone asking the questions 
rather than a form - better as you are more flexible than a set form ... forms can't talk back or adapt". Another was pleased not to be preached to "Thought you might go off on a moral tangent about having risky sex. Glad you didn't". Several noted the opportunity to think about sex in a different, deeper way "it did make me think a bit more about what I do sexwise and why I do it. It opened my eyes to what I actually do. I haven't discussed it on this level before".

Many participants also expressed their gladness to have been involved with the research, and several hoped that they had been helpful and that the research would be successful "I hope it may have been useful to you to read what I had to say with regard to your research. I hope you are able to find some factor or factors or lack of commonality to produce a report". Superordinate Theme: Technological issues

This theme focuses on technological competency, data security and anonymity, and the effect of the absence of non-verbal cues, particularly on the researcher's interview style and participants' ability to be open and honest.

Some issues with the chat software were commented upon by participants. One who was unable to make the secure software work commented "there's a level I obviously cant comprehend, whoever said the net was easy - lied!" Participants expressed no concerns about the security of the software or confidentiality generally. Two participants who were unable to use the secure software due to compatibility issues were unconcerned at the possibility of using less secure software to conduct the interview; one said "I know the risks of using MSN, and don't care, it's fine by me".

This lack of concern about the security of the software can perhaps be best understood in the context of anonymity issues. Several participants remarked that they found typing easier than talking in person, valuing the anonymity provided by the research paradigm. One participant 
clarified that their identity would be changed, but several had provided a false name to solve this issue themselves “I don't care about anonymous you only know my screen name”.

The software had a facility that allowed one person to see when the other was typing which enabled the dialogue to proceed in a largely ordered fashion and comprehensibility was not impaired in this way. There were times when both participant and researcher "spoke" at once, but no more frequently than naturally occurs in spoken conversations. Some participants were more laconic than others, one remarking that their interview "only took 50 minutes" and another apologizing for his "advanced hunt and peck" typing. However, several participants were able to provide large amounts of text with minimal prompting by the researcher, one interview notably continued for 3 hours, producing 359 lines of transcribed text. While this volume is considerably less than for spoken interviews, transcripts had a particularly "condensed" feel about them and often one line of text contained two or more emergent themes, as can be seen from the quotations used to illustrate the analysis.

Importantly, the use of internet interview procedures appeared to enable participants to be more open and honest than would be possible in face to face interviews. Participants commented that they were able to be honest both in the words they used and in their discussions, and this candidness was obvious in their transcripts. One commented that the interview was "like lots of the chats I do on msn with guys", indicating that his familiarity with online communication, especially for their discussions about sex, increased his comfort with engaging in this research topic. Another participant said that he found the Internet "easier than talking in person. i am more able to be honest and open".

Both participants and researcher were aware of the lack of availability of eye contact, body language and other non-verbal cues using this methodology, in contrast to face to face 
research interviews. During the debrief, one participant asked "As a researcher is the internet so impersonal that you loose the input from body language, creates a bias?" At a surface level, this lack of non-verbal cues did not appear to present great difficulty to either the researcher or participants as all were experienced in communicating using text-based instant messaging. However, on three occasions, it was possible to discern a sense of hostility in participants' text that had a different feel to the rest of the interview and at these times it was productive to comment on the possibility that the participant was offended. This can be seen in the context of one participant's talk about discussing condom (non)use with his partners on the Internet:

[I] if we have met on the net we may have talked about it ... if not then it may be talked about at another point. i dont just meet for getting fucked bareback. if he asked about it $\mathrm{i}$ wil tel him $\mathrm{i}$ do it

[R] ok. sorry if i offended you ... it's a bit difficult to say what i mean on here :)

[I] dont worry about. u cant se my body language or my eyes this way

This is also present for another participant describing how he thinks about the risks involved with barebacking:

[R] are there other reasons that you bareback?

[D] i like cum in me!

[R] yeah, i think you mentioned that

[D] i am not 'chasing' per se

[R] okay 
[R] tell me about that

[D] i am not actively seeking hiv

[R] but?

[D] i accept the risk

[R] how do you feel about the risk?

[D] it is something $\mathrm{i}$ have to accept cos $\mathrm{i}$ bb. cant pretend to be innocent

[R] so the risk is worth it?

[D] that is a question that has to go unanswered. you can stil get an std using rubbers

[R] did my question offend you?

[D] yes

[R] i'm sorry. it'd help to know how.

[D] i'm not stupid; i choose to bb. we all accept risk in our lives. we do risky thing because of the thrill

During the debrief, we talked about whether he felt he needed any follow-up after the interview:

[R] is there anything you feel you need from the 'medical' community - in terms of info, advice, therapy, etc?

[D] nope. npow that does offend me

The above examples demonstrate that the researcher needs to be experienced in internet communication in order to receive and work with the subtle nuances that are present in dialogue 
of this form. We also felt that a working knowledge of some of the abbreviations (topic specific and otherwise) that are used in online chat is also needed in order that the researcher is able to progress beyond clarification into a higher level of engagement with the interview. However, we recognize that this makes the process of neutrality and "bracketing off" of the self when conducting interviews and analyses within phenomenological approaches more complex.

\section{Superordinate Theme:Boundaries}

This theme concentrates on participants' interactions with the researcher as flirtatious, intimate and curious about the researcher's sexual preferences. It also explores intrusions to the research non-physical "space".

There were a considerable number of instances where participants used language that was overtly flirtatious or otherwise intimate. This ranged from the relatively innocuous insertion of a kiss "ok bye $\mathrm{x}$ " at the end of an otherwise unremarkable interview, to particularly overt attempts at engagement beyond the boundaries of the research interview. Two participants were clearly attempting to engage with the researcher in a different persona. Here, one comments that a British researcher is likely to be uncircumcised ("uc"), a fantasy that is clearly attractive to him: "I was aiming to charm. And after your study is over, if ever in NYC, we can have a cuppa. Course I have I have a soft spot for brits (and yeh I have preference for uc, so I gave myself a little push for cooperation w/a [likely uncut] psychologist)". Other participants were more subtle "anything you want to ask that wasn't on your question paper". Whilst it might be argued that participants might be applying their usual "internet self" to the interview, this was in the full knowledge that flirtation was not entirely appropriate conduct for such an interview as one participant replied when I was checking that he had read the participant information "yeah, you ask questions, and I dont flirt". Another said "sorry if ive been a bit pushy". 
Some participants were particularly keen to ask questions about the interviewer's sexual feelings and preferences, three clearly wanted to know whether he barebacked. The strongest example of participants' thinking about this was "So where do youcome out? Hav you indulged? I interpret this is a personal concern for you, w/o knowing if you do or dont bb would be a litle disappointed if you were horrified by bb". When describing sexual activities that he enjoyed, this participant asked "Yep - get you hot? <eg>". The slower pace of Internet interviews allowed the researcher to respond in a considered manner and refer participants to the participant information which stated that the researcher would not answer questions about his own sexual experiences or preferred activities. Participants did not seem to find such a response particularly difficult, and while some were clearly aware that they were pushing the boundary (and continued to do so), for others this seemed less intended and they made no further attempts to elicit personal details about the researcher. In all cases, the interview was able to proceed satisfactorily.

The lack of a physical shared space allowed several other factors to enter that may be different from the usual face-to-face research setting. On asking one participant whether he was ready to begin, he replied "well i might watch the tv at the same time ...". Several longer-thanusual delays were explained by participants who "just need to get a cuppa", "just had to piss" and "sorry about the delay, pizza arrived". One participant terminated the interview early, despite a clear request to allow 60 to 90 minutes, to "meet a real estate agent ... to purchase another property".

Superordinate Theme:Requests / Expectations 
This theme includes participants' uncertainty about this research and Internet qualitative research more generally, the need to be "led" by questions, and the possibilities around the researcher "feeding back".

Some of the participants seemed unsure what to expect from a qualitative interview and expressed this most obviously at the beginning of the interview. Some asked "where do we start?" or "how do we do this" which may be interpreted as a request for the researcher to take a more active lead in the research. However, as these questions typically occurred after saying hello, this provided a useful opportunity to check that participants had read the information and to revisit consent. This was necessary as despite only allowing participants to sign-up for the study once they had read the participant information, and checking that they had done so at the start of the interview, some participants had clearly missed or forgotten important information contained therein. For example, several participants asked "are you gay?" and once "are you a shrink?”, despite information on the first author's sexual orientation and occupation being provided within the participant information.

Others were more explicit about their need to be led in the interview, or their expectation that this should happen. One participant commented "it is your interview hehe im just the test subjet" and another, when telling me to ask more questions, said "I had 15 yrs of good interpersonal therapy, I know when I'm being drawn out to keep talking”. This is a clear reflection of the conflict between trying to say the minimum necessary to keep the conversation flowing, and participants feeling uncomfortable with such an approach, especially in the lack of the usual verbal and non-verbal cues that the researcher is listening.

Several participants were keen to know more about the research. These questions varied from a simple clarification of the research question and requests that the research should be 
published in order to "see it on the net, all the work put together to see what is in common", to more advanced questions about the methodology. One participant in particular was keen to discuss the difficulties of how it would be possible to "compile anything" from a series of interviews, the role of objectivity and "huge potential for unconscious bias" with the interpretation used in the analysis. This suggests that some participants used to questionnaires and experiments are unaware of the purpose, reliability and validity of qualitative methodologies and may benefit from being directed to resources that explore these issues further.

Finally, participants were ambivalent about receiving feedback on their process. All participants described their involvement in the research as a positive experience, some going as far as to recognize that the interview allowed them to talk about and process sex in a way that was new to them. However, when asked in the debrief whether they felt they needed any form of follow-up intervention such as advice, information or therapy, all participants declined. Despite this, one participant repeatedly asked "whats your take on me" and several others sought validation of their perspective or experience in less direct ways, such as asking in the debrief “did i shock u?". Regardless of the benefit that some found from the interview and their keenness for feedback, most were quick to say that they were just "not really the type for therapy" which perhaps says as much about gay men's beliefs and experiences of sex therapy as it does their desire to further explore sexual issues.

\section{Discussion}

The current study's innovative use of IPA and Internet chat elicited rich dialogues, permitting insights into gay men's experience of barebacking and Internet sex-seeking from participants who would not have participated in face-to-face qualitative research. However, the effect of this method of interviewing on participant and researcher discourses is largely unknown 
and is worthy of ongoing research. In this study, we aimed to share some reflections on this process in order to further develop and evaluate the methodology.

Participants were overwhelmingly positive in their evaluation of the research interview. They enjoyed the space, finding it helpful to talk about an issue that they had never been able to discuss at such a deep level before. This supports the observations of several researchers that Internet users appreciate the familiarity, comfort and safety of an online interview (e.g. Mann \& Stewart, 2000), which permits a relaxed and informal atmosphere (e.g. Madge \& O’Connor, 2004), and allows for sensemaking and giving something back to the community (e.g. Beck, 2005).

The degree of difficulty that some experienced computer users had in installing and operating the secure software was problematic. This is a common problem (Illingworth, 2001; O'Connor \& Madge, 2001) and highlights the importance of both the researcher and participant having the necessary level of technical expertise to operate and troubleshoot the software that is used, or that both parties have access to appropriate technical support, perhaps supplied by the researcher's host institution.

The degree to which participants in the current study seemed unconcerned about data security and confidentiality, despite considerable information about the potential risks, might indicate that software providing the highest degree of security (including encryption) may not need to be provided as standard. Participants might prefer to choose the level of security they require, possibly preferring chat software that they are already familiar with and are competent in using. It is also important to note that participants in the current study were used to talking about and negotiating sex online, and therefore may have a greater tolerance for the potential risks of an online research interview being intercepted than the general population. However, it is 
incumbent upon investigators to work to assure the privacy and safety of participants, even if they themselves seem unconcerned about the risks of their participation.

Participants particularly enjoyed the anonymity of the research interview, which is consistent with the findings of Hewson, Yule, Laurent, and Vogel (2003). We suggest that anonymity can be important in discussing embarrassing and sensitive topics, although we acknowledge that the inability to verify the identity of the participant (and the researcher) may be problematic. However, we feel that the need for verification of the participant's identity is questionable and may depend on the aims of the particular research. In face-to-face interviews for interpretative phenomenological analysis, one is equally unable to verify that a particular participant has experience of the topic in question. Furthermore, the slower pace of the interview, alongside the ease of "logging off" the Internet (as opposed to leaving an interview room) may also make it more likely that "imposters" would terminate the interview prematurely. Equally, it seems unlikely that a participant would be able to sustain an interview on a subject of which they have no experience, for ninety minutes to two hours, without the researcher becoming aware of this. Researchers using other research methodologies and epistemologies that are more reliant on the concept of verifiable, objective "truth" may have differing views on this issue. For example, Taylor (1999) asserts that acceptance of "online life as a thing in itself" is important, and Valentine (2001) believes that online textual personas cannot be separated from the offline physical person who constructs them and they are commonly based on offline identities in any case.

One of the major criticisms of online interviews has been that the typed exchange does not approximate to transcriptions of verbal interviews, primarily through inability or difficulty in the use and interpretation of paralinguistic communication (Chen \& Hinton, 1999). Online 
interviews and their transcripts have been described as short, closed, staccato, pointed, nonlinear, ambiguous, slow, using acronyms and abbreviations (Davis et al., 2004). These authors also suggest that participants "edit out" aspects of their experience, leading these authors to conclude that the knowledge derived from the online interviews was somewhat limited. It would be foolish (and untrue) to argue that online interviews are none of these things. However, our experience was that many of these deficiencies can be ameliorated by the researcher's experience of online communication, ability to type quickly, and knowledge or experience of the topic they are researching. Furthermore, in accordance with Madge \& O'Connor (2004), who found participants" material to be "less inhibited, more direct and less likely to edit thoughts to give socially desirable answers", we found that it was common for participants to reveal very personal and sensitive information that they would not feel able to reveal in a face-to-face interview. This highlights the need to treat the participants' data as valuable in their own right, and to celebrate the advantages and complimentary nature of the methodology, rather than finding it deficient in comparison with other methodologies. We feel that it is particularly valuable, when investigating a phenomenon that is highly integrated with Internet communication (such as gay men's Internet sex-seeking), to allow participants to use the same mode of communicating in the interview, as they do for their "real-life" encounters.

Our participants, although of different ages, were all familiar with computers and online communication and had access to a private computer, and we recognize that other populations, particularly older, technologically inexperienced individuals, may feel less comfortable using this particular medium for discussing highly personal subjects.

We noted that the typing skill of some participants led to a reduced output, but this is not necessarily a problem in qualitative methods where emergent themes are compared within and 
between participants - for many themes that were under explored by some participants, others provided much more detailed accounts. We were prepared to have more "question and answer" type exchanges and had produced a number of prompts for use in the semi-structured interview, where necessary. In agreement with Davis et al. (2004), we found that the slower nature of the interviews allowed participants' responses to be more processed and thoughtful, leading to a more condensed transcript.

The lack of body language, and features of spoken language such as emphasis, pitch, tone, non-words such as "umm", “errr", unfinished sentences and the like may be problematic for researchers using methodologies that rely heavily on these, such as conversational analysis. However, Seymour (2007, p. 1194) comments that "we don't just leave the body behind as we enter cyberspace, and 'real life' isn't somehow automatically more 'real' than 'virtual' experience”. Similarly, Madge \& O’Connor (2004) assert that mannered behavior, preinterpreted meanings and unstated assumptions are visible in the online interactions and available for analysis. These authors also commented that they found themselves "lost for words" at times, when they worried that any comment they might have wanted to make felt banal, and questions that they considered asking felt leading. They reflected on the need for "explicit empathy rather than utterance and gesture" in order to transcend this problem, and we found on many occasions that the use of a curious, reflective and somewhat interpretative interview style was sometimes necessary but always sufficient to provide rich and lengthy interactions. This level of researcher involvement during the interview does somewhat conflict with the principles of epoché (the bracketing off of one's own feelings and experience) and neutrality. However, in common with interviewing people with learning disability (where verbal output can be equally reduced) using IPA (e.g. Wheeler, 2007), a more reflective and interpretative style to the 
interview, and certainly to the analysis, is perhaps justifiable. A methodology such as grounded theory, which is able to make use of previous interviews in order to structure successive transcripts (as well as the requirement for purposive sampling, which is often difficult when relying on face-to-face interviews about a relatively rare experience or sensitive topic) might be relatively better suited to online interviewing.

The lack of a shared physical space allowed interview boundaries to be easily transgressed by participants. It was common for participants to flirt, request contact outside of the research boundary, and seek personal information about the interviewer's sexual feelings, preferences and behaviors. This behavior occurred in the context of the interviewer disclosing his sexuality in the participant information, whilst stating that attempts to engage the researcher in conversations that were not relevant to the research, or about his own sexual preferences, were not appropriate. While this phenomenon was likely exaggerated by the topic being discussed, it appears that such dynamics might increase in frequency and intensity when interviews are conducted on the Internet. Some authors suggest that online participants are more likely to divulge personal information about themselves if the interviewer initiates the disclosure process (e.g. Curasi, 2001) and to compensate for lack of visual cues to facilitate rapport, Madge \& O'Connor (2004) initiated a personal relationship in preparation of the interviews. While an enhanced research relationship may indeed be permitted by contact and appropriate disclosure before the interview, we suggest that researchers do so with caution. In agreement with Dickson-Smith, James, Kippen and Liamputtong (2008), given the potential for blurred boundaries, we recommend the use of appropriate supervision and reflexivity to consider the message that participants may receive from sensitive online interactions, and to balance the potential for an enhanced rapport with the risk of being in a compromising situation in which 
there might be accusations of improper behavior. However, the degree to which participants tested the research boundary was also suggestive of a high level of comfort with the interview setting. We reflected that participants were very used to sitting at home, on the Internet, talking to a gay man about sex, and found it very easy to do so with another, who happened to be a researcher. This suggests a very high degree of ecological validity in the data collected.

Several recommendations can be drawn from the experience of conducting research using the Internet. We would advise researchers contemplating using the Internet for online interviews to consider where their personal and professional boundaries lie in terms of participants attempting to engage with them at a personal level; how they communicate these boundaries before and during the interview; and how they will use research and personal supervision in order to process the feelings that are generated by participants' attempts to engage them in this manner. The researcher must be aware of and prepared to manage unwelcome intrusions into the interview space, which is of course the participant's (and perhaps the researcher's) home. These may include requesting that participants turn off the television and their mobile telephone; close down all programs apart from the chat software used for the interview; and otherwise prepare for the interview in a way that will minimize interruption (such as going to the toilet and fetching a glass of water). Interviews lasting in excess of one hour, however, would be quite reasonably punctuated by a break or two, at the very least to allow participants a few minutes away from the screen.

Participants sometimes seemed unsure what to expect from a qualitative interview and seemed unsure of the value or validity of qualitative research generally. We recommend that researchers use the participant information and/or the start of the interview to provide information about the difference between quantitative and qualitative paradigms, a brief 
explanation of how qualitative analyses are performed and validated, perhaps directing interested participants to appropriate Internet resources. We also noted that participants were unanimous in their desire to read the results, and we recommend that this opportunity be offered to all participants. This might also be a useful way of deflecting participants' requests of the researcher for more psychotherapeutic "insights" into or interpretations of their behavior or personality.

It might also be useful for the researcher to consider, and perhaps share with the participant, something about their expectations of how much they intend to speak, and how much reflecting, probing and interpretation they are willing to provide. Our experience that some participants had difficulty with directing the conversation and maintaining conversational flow, and were unwilling to do so is important in the context of the question posed by Hamilton and Bowers (2006, p. 832), namely whether online interviews are "more egalitarian and thus shift the 'expert' role more to the participants and away from the professional" .

Several participants had either forgotten, not read, or misunderstood elements of the participant information, and we recommend that in cases where researchers cannot observe participants' reading of this information, that any particularly salient points (such as boundaries, disclosure, etc) are restated at the beginning of the interview.

\section{Conclusion}

Participants and researchers found the internet-based IPA paradigm to be largely suitable for interviewing gay men about their experiences of Internet sex-seeking and barebacking, for analysis with IPA. Participants appreciated the advantages of Internet interviews (particularly the anonymity) and felt the experience was both enjoyable and useful in allowing them to talk about material in depth, in a way that they did not feel would be possible in face-to-face 
interviews. There were minor issues with technical expertise and software compatibility, but these were well tolerated by participants who felt comfortable using less secure software. The ability of participants to make informed choices about the level of anonymity and data security they require (within ethically sound limits) may currently be underestimated by researchers and should be explored further. Issues surrounding the verification of participant identity were not found to be problematic in the current study. The style of interaction, particularly a more condensed, slowed-down exchange with few non-verbal cues, may be challenging for researchers who are unused to communicating in real-time on the Internet, or who are not familiar with terminology used by participants.

The current research contrasts with the assertions of Davis et al. (2004) that "online synchronous interviews do not readily lend themselves to the exploration of meaning, raising questions about how they can contribute to an in-depth description of social or sexual experience". We found that Internet interviews do permit rich and useful data, and that the adaptation of interview style to one that is more interpretative, can ameliorate some of the above difficulties, although we recognize that this may be a poor fit with some epistemologies and methodological approaches to qualitative analysis. We noted a high potential for boundary transgressions, in both interruptions to the research, and requests for intimate and flirtatious interactions. This was alongside a sometimes poor understanding on the part of the participant, of the "norm" for qualitative interviews and analysis. The anonymous nature of the Internet appears to give greater potential for lack of clarity on these issues, especially when investigating behaviors and feelings using a medium that allows, and is commonly used, for more intimate talk. Detailed attention to preparation that elicits and clarifies participant (and researcher) expectations of boundaries and aims of the research is necessary to manage opportunities where 
these may be in conflict. Countertransference, in the form of feelings evoked by the interviewee in the interviewer, is equally relevant to clinical research as it is to practice, and should not be underestimated in Internet research.

In summary, the current research indicates that online qualitative research is valuable in its own right, and that the advantages considerably outweigh the difficulties, particularly in accessing individuals who may not present to other research settings, to discuss experiences that would otherwise be too difficult to talk about. Several issues that are problematic in more conventional research still apply, albeit in a different guise, but these must be situated in the context of who is being researched, what and why, and can be largely overcome with more creative methodologies and considered preparation. Further accounts of transferential, technological and analytic issues within internet qualitative methodologies remain necessary and we await them eagerly.

\section{Endnotes}

${ }^{1}$ For the purposes of this study, we define barebacking as intentional unprotected anal intercourse (UAI) between individuals who are not primary partners in a negotiated safety arrangement. Negotiated safety is where two men in a relationship decide not to use condoms when having sex with each other. It relies on them having the same HIV status. Both present for two HIV tests, three months apart, avoiding UAI in the interim. If both have the same HIV status at the second test, they stop using condoms within their relationship but keep using condoms for risky sex outside of that relationship.

${ }^{2}$ Gay Times is a lifestyle magazine targeted at gay men.

${ }^{3}$ The websites used were outeverywhere.com, thingbox.co.uk, kagoul.co.uk and bareback.com. Individuals registering with these websites can build online "profiles" including pictures of 
themselves and personal information, with the aim to talk online with, and often meet, interested others.

\section{References}

Beck, C. T. (2005). Benefits of participating in internet interviews: Women helping women. Qualitative Health Research, 15, 411-422.

Chen, P. \& Hinton, S. M. (1999). Realtime interviewing using the World Wide Web. Sociological Research Online, 4(3). Retrieved April 19, 2007, from http://www.socresonline.org.uk/4/3/chen.html

Chen, S. S. \& Hall, J. (2003). Online social research: Methods, issues and ethics. New York: Peter Lang.

Coombes, H. (2001). Research using IT. Hampshire: Palgrave.

Curasi, C. F. (2001). A critical exploration of face-to-face interviewing versus computermediated interviewing. International Journal of Market Research, 43(4), 361-375.

Davis, M., Bolding, G., Hart, G., Sherr, L. \& Elford, J. (2004). Reflecting on the experience of interviewing online: Perspectives from the internet and HIV study in London. AIDS Care, 16(8), 944-952.

Dickson-Smith, V., James, E. L., Kippen, S. \& Liamputtong, P. (2008). Risk to researchers in qualitative research on sensitive topics: issues and strategies. Qualitative Health Research, 18, 133-144.

Fox, F. E., Morris, M. \& Rumsey, N. (2007). Doing synchronous online focus groups with young people: Methodological reflections. Qualitative Health Research, 17, 539-547.

Hamilton, R. J. \& Bowers, B. J. (2006). Internet recruitment and e-mail interviews in qualitative studies. Qualitative Health Research, 16, 821-835. 
Hewson, C., Yule, P., Laurent, D. \& Vogel, C. (2003). Internet research methods. London: Sage. Hunt, N. \& McHale, S. (2007). A practical guide to the e-mail interview. Qualitative Health Research, 17, 1415-1421.

Illingworth, N. (2001). The internet matters: exploring the use of the internet as a research tool. Sociological Research Online, 6(2). Retrieved April 19, 2007, from http://www.socresonline.org.uk/6/2/illingworth.html

Jones, S. (1999). Doing internet research: Critical issues and methods for examining the net. London: Sage.

Madge, C. \& O'Connor, H. (2004). Exploring the internet as a medium for research: Web-based questionnaires and online synchronous interviews. Retrieved April 19, 2007, from http://www.ccsr.ac.uk/methods/publications/documents/WorkingPaper9.pdf

Mann, C. \& Stewart, F. (2000). Internet communication and qualitative research. London: Sage. O’Connor, H. (2006). Online interviews module. Exploring online research methods in a virtual training environment. Retrieved April 19, 2007 from http://www.geog.le.ac.uk/orm/interviews/intcontents.htm

O’Connor, H. \& Madge, C. (2001). Cybermothers: Online synchronous interviewing using conferencing software. Sociological Research Online, 5(4). Retrieved April 19, 2007, from http://www.socresonline.org.uk/5/4/oconnor.html

Seymour, W. (2007). Exhuming the body: Revisiting the role of the visible body in ethnographic research. Qualitative Health Research, 17, 1188-1197.

Taylor, T. L. (1999). Life in virtual worlds: Plural existence, multimodalities and other online research challenges. American Behavioral Scientist, 43, 436-449.

Valentine, G. (2001). Social geographies: Space and society. Harlow: Prentice Hall. 
Wheeler, P. (2007). "I count myself as normal, well, not normal, but normal enough": Men with learning disabilities tell their stories about sexuality and sexual identity. Tizard Learning Disability Review, 12(1), 16-27.

Russel Ayling, MA(Hons), DClinPsy, is a clinical psychologist working in HIV and sexual health at Barts and the London NHS Trust, in London, UK.

Avril J Mewse, BA(Hons), $\mathrm{PhD}$, is a senior lecturer in the School of Psychology at the University of Exeter, in Devon, UK. 